\title{
ChemComm
}

\section{Membrane destabilization by monomeric hIAPP observed by imaging fluorescence correlation}

49, 9155

Received 29th June 2013,

Accepted 5th August 2013

DOI: $10.1039 /$ c3cc44880k

www.rsc.org/chemcomm

Monomeric hIAPP significantly destabilizes both model and live cell membranes by increasing membrane fluidity. This interaction with membranes happens via carpet formation followed by lipid extraction in a concentration dependent manner and thus we propose that hIAPP aggregation prior to membrane interaction may not be necessary for its cytotoxicity.

Membrane association of aggregation-prone proteins and peptides has emerged as a critical factor in numerous degenerative diseases. In the last two decades, human Islet Amyloid Polypeptide (hIAPP) was shown to be an important amyloidogenic peptide implicated in progressive pancreatic degeneration in diabetes mellitus type II. hIAPP is a 37-residue peptide hormone that is co-packaged and co-secreted with insulin upon glucose stimulus and has been found to regulate physiological processes viz. gastric emptying and regulation of insulin response. ${ }^{1}$ Of particular interest is the observation that in vitro aggregation of hIAPP causes membrane damage by a 'pore formation' mechanism that is believed to cause cytotoxicity. ${ }^{2}$ A concern in these assays, however, is the high concentration of hIAPP used (10-40 $\mu \mathrm{M})$ at which it aggregates; while physiological hIAPP exists as a monomer at a concentration of 20-100 pM. ${ }^{1}$ Thus, it is imperative to investigate the mode of interaction of monomeric hIAPP and its effect on membranes in an attempt to discover the identity of the toxic entity in the case of pancreatic degeneration.

In this work, we used membrane dynamics as a metric to probe monomeric hIAPP induced membrane perturbation. We explored the effect of monomeric hIAPP on the fluidity of different supported lipid bilayers (SLBs) and live cell membranes using single molecule sensitive imaging total internal reflection-fluorescence correlation

\footnotetext{
${ }^{a}$ Departments of Biological Sciences and Chemistry and NUS Centre for Bio-Imaging Sciences (CBIS), National University of Singapore, 117557, Singapore. E-mail: twohland@nus.edu.sg; Fax: +65-6776 7882; Tel: +65-6516 1248

${ }^{b}$ Malaria Research Group, International Centre for Genetic Engineering and Biotechnology, Aruna Asaf Ali Marg, New Delhi, 110067, India.

E-mail: aseem@icgeb.res.in

$\dagger$ Electronic supplementary information (ESI) available: Experimental procedures, supporting figures, imaging video, hIAPP synthesis and characterization. See DOI: $10.1039 / \mathrm{c} 3 \mathrm{cc} 44880 \mathrm{k}$
}

spectroscopy (ITIR-FCS) ${ }^{3,4}$ and provide a mechanism of the hIAPPmembrane interaction. The detailed methods are given in the ESI. $\dagger$

We first establish that hIAPP exists as a monomer at $1 \mu \mathrm{M}$ and lower concentrations by a variety of methods. We used confocal FCS to measure the diffusion time $\left(\tau_{\mathrm{D}}\right)$ of Atto-R6G labelled hIAPP (AR6G-hIAPP). FCS can distinguish monomers and aggregates based on their respective diffusion times. The $\tau_{\mathrm{D}}$ of AR6G-hIAPP was $66 \pm 0.6 \mu \mathrm{s}$, which corresponds to the diffusion coefficient $(D)=311 \pm 5 \mu \mathrm{m}^{2} \mathrm{~s}^{-1}$ at concentrations of $40 \mathrm{nM}$ and $1 \mu \mathrm{M}$ (blue and green autocorrelation functions (ACFs) in Fig. 1A respectively). The obtained value of $D$ is in good agreement with the previous reports for monomeric hIAPP as measured by confocal FCS and diffusion-NMR experiments. None of the temporal intensity traces of 10 independent measurements on AR6G-hIAPP at each of these concentrations showed any
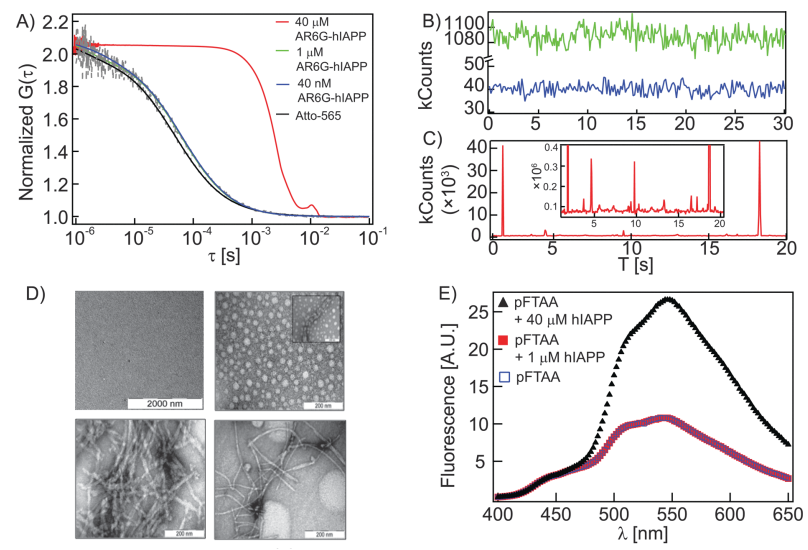

Fig. 1 Aggregation state of hIAPP. (A) ACFs of AR6G-hIAPP at different concentrations. (B) Intensity traces of $40 \mathrm{nM}$ (blue) and $1 \mu \mathrm{M}$ (green) AR6GhIAPP show no spike indicating the absence of aggregates. (C) Intensity trace of $40 \mu \mathrm{M}$ (red) AR6G-hIAPP shows a number of spikes indicating the presence of aggregates (the inset shows the same trace with a magnified $y$-axis showing a wide range of different intensity peaks). (D) TEM images of monomeric ( $1 \mu \mathrm{M})$ hIAPP (top left) and the oligomers (top right), protofibrils (bottom left) and fibrils (bottom right) of hIAPP. (E) The fluorescence spectra of the aggregation sensing dye pFTAA in the absence (blue open square) and in the presence of $1 \mu \mathrm{M}$ (red close square) and $40 \mu \mathrm{M}$ (black triangle) unlabelled hIAPP. 
intensity spikes supporting the absence of aggregates (Fig. 1B). However, intensity traces of $40 \mu \mathrm{M}$ hIAPP contain spikes indicative of aggregates (Fig. 1C). The existence of aggregates is confirmed by the right shift of the ACF as aggregates diffuse much slower than the monomeric species (Fig. 1A, red). Next, the TEM image of $1 \mu \mathrm{M}$ hIAPP did not show any feature (Fig. 1D, top left). For comparison, TEM images of oligomers (top right), protofibrils (bottom left) and fibrils (bottom right) of hIAPP are shown in Fig. 1D. A novel penta-thiophene dye, pFTAA, that selectively binds to amyloidogenic oligomers ${ }^{7}$ did not show any increase in its fluorescence intensity (Fig. 1E) nor a change in its absorption spectra (Fig. S1A, ESI $\dagger$ ) upon addition of $1 \mu \mathrm{M}$ unlabeled hIAPP suggesting that hIAPP did not form oligomers. The conventional thioflavin $\mathrm{T}$ assay also did not show any fibril formation at this concentration (Fig. S1B, ESI $\dagger$ ). The CD spectra of hIAPP at $1 \mu \mathrm{M}$ concentration (Fig. S1C, ESI, $\uparrow$ red) exhibited a dominant helical component suggesting the absence of fibrils. At $40 \mu \mathrm{M}$ hIAPP aggregated into amyloid fibres (Fig. S1D, $\mathrm{ESI} \dagger$ ) with $\beta$-sheet conformation (Fig. S1C, ESI, $\uparrow$ black). Taken together, these observations suggest that hIAPP remains monomeric in the range of concentrations ( $40 \mathrm{nM}-1 \mu \mathrm{M})$ used for this study.

Interaction of unlabelled hIAPP with the rhodamine $\mathrm{PE}$ (RhoPE) labelled SLBs was studied by measuring the lateral diffusion coefficient $(D)$ of the latter at $310 \mathrm{~K}$. Membrane diffusion is a potent reporter of its integrity. The $D$ of the DOPC : DPPC $(1: 1)$ SLB which exhibits gel/fluid phase co-existence increased in a time dependent manner. The $D$ increased from $3.0 \pm 0.4 \mu \mathrm{m}^{2} \mathrm{~s}^{-1}$ to $5.8 \pm 1.2 \mu \mathrm{m}^{2} \mathrm{~s}^{-1}$ (at $40 \mathrm{~min}$ ) (Fig. 2A, green). For the same concentration of peptide, an SLB composed of DOPC : DPPC : Chol (1:1:1), a canonical 'raft mixture', exhibited a $70 \%$ increase in $D$ (Fig. 2A, red). An SLB composed of DOPC: Chol (5:1), however, showed only $40 \%$ increase in $D$ (Fig. 2A, blue) whereas hIAPP had little effect on a pure DOPC SLB (Fig. 2A, black). We also observed an almost instantaneous formation of fluorescent structures in
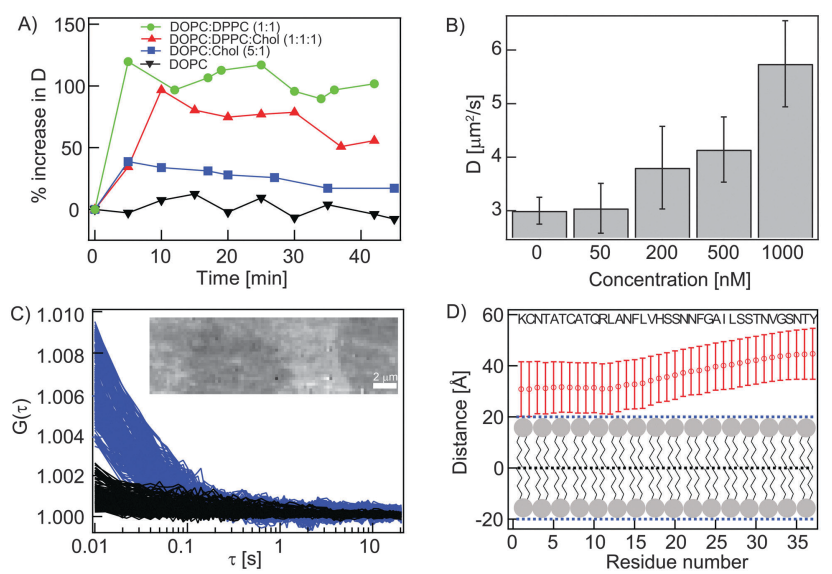

Fig. 2 Interaction of monomeric hIAPP with SLBs. (A) Percentage increase in D of different RhoPE labelled SLBs upon treatment with unlabelled hIAPP (1 $\mu \mathrm{M})$. (B) hIAPP concentration dependent increase in $D$ of the RhoPE labelled DOPC: DPPC (1:1) bilayer. (C) ACFs of the diffusing 'carpet' of AR6G-hIAPP (blue, corresponds to the bright region of the TIRF image shown in the inset) and background (black, corresponds to the dark region of the same image). In the inset, the TIRF image shows that AR6G-hIAPP carpets only form on the unlabelled DOPC SLB top (bright), not on the non-membrane region (dark). (D) The predicted orientation of hIAPP over a membrane by MCPep shows peptide insertion to be unfavorable. the different bilayers upon addition of the peptide (Video 1, ESI $\dagger$ ). As these structures were brightly fluorescent, we infer that they contain a large number of lipid molecules extracted from the RhoPE labelled SLB along with the non-labeled peptide as a non-specific hetero-complex. Though a similar process has been proposed earlier, we present experimental evidence that the extraction of lipid affects its stability. ${ }^{8}$ The observed increase in membrane fluidity by lipid extraction is inversely correlated to the 'free area' of the membrane (DOPC > DOPC : Cholesterol > DOPC : DPPC : Chol > DOPC : DPPC). Lipid extraction from a gel lipid containing SLBs causes much larger release of free area compared to that of fluid SLBs and thus a dramatic change in $D$ was observed for DOPC : DPPC SLBs.

The increase in membrane fluidity was also dependent on the peptide concentration used (Fig. 2B). Low concentration of unlabelled hIAPP (50 nM) did not cause any change in $D$ of the RhoPE labelled DOPC: DPPC $(1: 1)$ bilayer $\left(3.0 \pm 0.4 \mu \mathrm{m}^{2} \mathrm{~s}^{-1}\right.$ at $310 \mathrm{~K}$ ) suggesting that physiological levels of hIAPP would be non-damaging to cellular membranes. However, at moderately higher concentrations (200-500 nM) we found that the peptide increased fluidity. Higher peptide concentrations $(1 \mu \mathrm{M})$ resulted in a still higher increase in membrane fluidity $\left(D=5.8 \pm 1.2 \mu \mathrm{m}^{2} \mathrm{~s}^{-1}\right)$. Though intuitive, we demonstrate here that the extraction of lipids from the bilayer by sub-micromolar hIAPP concentrations results directly in the increase in membrane fluidity and not necessarily collapse.

Nevertheless, hIAPP has to interact with the SLB to be able to extract lipid. To probe the association of the peptide with the SLB, we added non-damaging concentration of AR6G-hIAPP (50 nM) to an unlabelled SLB and imaged the system using total internal reflection fluorescence (TIRF) microscopy. TIRF videography showed the peptide to form a diffusing carpet-like structure over the membrane (Fig. 2C and Video 2, ESI $\dagger$ ). The dark areas in the image (and in Video 2, ESI $\dagger$ ) are areas of membrane defect/collapse where AR6G-hIAPP does not form a carpet. The apparent diffusion coefficient of the peptide was computed to be $9.03 \pm 5.92 \mu \mathrm{m}^{2} \mathrm{~s}^{-1}$, which was roughly 3 times higher than the free diffusing lipid probe in the membrane suggesting no penetration of hIAPP (Fig. S2, ESI $\dagger$ ). Recent studies indicate that hIAPP can assume betasheet-like structures on artificial surfaces without forming higher order oligomers/fibres. ${ }^{9}$ Moreover, the predictions from the Monte Carlo simulation (using the MCpep server ${ }^{10}$ ) suggest that the insertion of hIAPP into the membrane is not energetically favourable (Fig. 2D and Fig. S3, ESI + ). hIAPP assumes an amphiphilic helical structure with two short hydrophobic stretches alternating with polar patches (Fig. S4, ESI + ), ${ }^{11}$ a conformation that may not favour its penetration into the membrane but facilitate surface orientation (as predicted) favoured by local electrostatics as in ALPS/class A2 domains. ${ }^{12}$

Monomeric hIAPP did not form pores in liposomes as observed from the confocal FCS based dye leakage experiments (the principle of the experiment is given in Fig. S5, ESI $\dagger$ ). The dye was not released from either rhodamine labelled vesicles (RLVs) or rhodamine entrapping vesicles (RLVs) upon treatment with $1 \mu \mathrm{M}$ of unlabelled monomeric hIAPP (Fig. S6A and B, ESI $\dagger$ ), rather the liposomes aggregated. As expected, hIAPP at aggregating concentration $(40 \mu \mathrm{M})$ caused dye release (Fig. S7, ESI $\dagger$ ). We therefore 

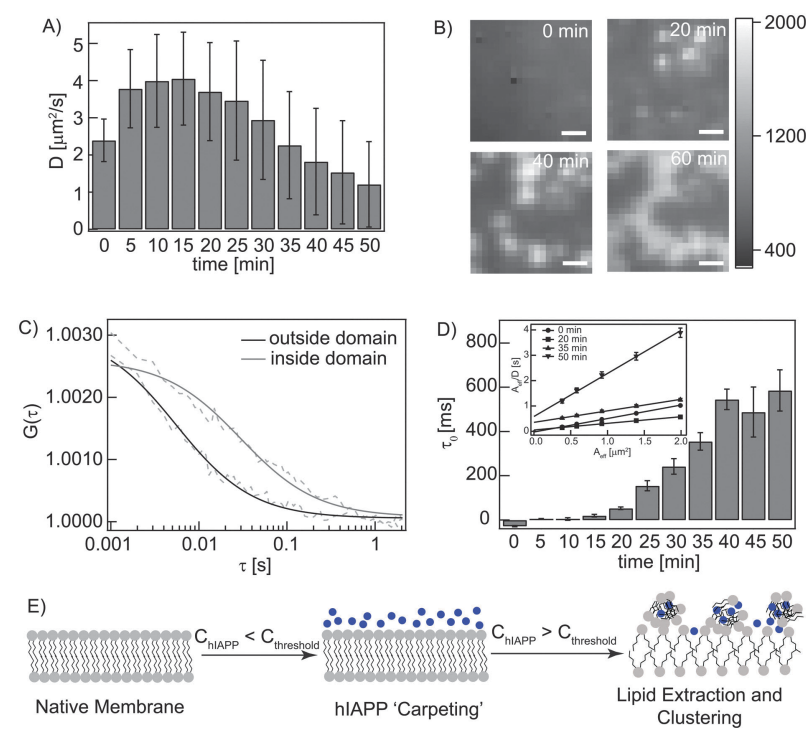

Fig. 3 Interaction of monomeric hIAPP with plasma membranes. (A) A time dependent increase and subsequent decrease in $D$ of Dil labeled plasma membrane on treatment with $1 \mu \mathrm{M}$ unlabelled hIAPP. (B) TIRF images of the lower membrane of hIAPP treated cells (at measured ROI) at different time points. The scale bar is $1 \mu \mathrm{m}$. (C) ACFs of the labelled membrane inside and outside the bright structures. (D) Time dependent increase in FCS diffusion law intercept $\left(\tau_{0}\right)$. The inset shows the diffusion law plots at representative time points. (E) Schematic representation of 'carpeting' and lipid extraction by monomeric hIAPP.

believe that monomeric hIAPP associates with the membrane following the 'carpet model' instead of the widely speculated 'pore model'. ${ }^{13}$

We next explored if hIAPP interacts with live cell membranes in a similar way. DiI-C ${ }_{18}$ labelled SH-SY5Y membranes in the absence of hIAPP showed a $D$ of $2.4 \pm 0.4 \mu \mathrm{m}^{2} \mathrm{~s}^{-1}$ at $310 \mathrm{~K}$ (Fig. 3A). Unlabelled hIAPP $(1 \mu \mathrm{M})$ was added to the cells, as this concentration was sufficient to increase membrane fluidity for a SLB. A time dependent increase in membrane fluidity $(D=$ $4.1 \pm 1.2 \mu \mathrm{m}^{2} \mathrm{~s}^{-1}$ ) in the initial $20 \mathrm{~min}$ was observed (Fig. 3A). Thereafter, brightly fluorescent plasma membrane derived structures were visible that exhibited an apparently slower $D$ (Fig. 3B and C). The standard deviation (SD) is a reflection of cell membrane heterogeneity and is in a typical range of $17-25 \%$ as seen by FCS measurements for the cell membrane. ${ }^{14}$ For measurements at later time points, the SD rises to $95 \%$ indicating an increase in heterogeneity after hIAPP interaction (see ESI $\dagger$ for details). Although difficult to ascertain, these structures might resemble vacuole like structures formed in $\beta$ cells upon hIAPP treatment that are associated with cellular degeneration. ${ }^{15}$ In our experiment, the number and size of these structures increased with time. We show a 'FCS movie' indicating temporal changes in spatial distribution of $D$ at a region of interest (ROI) with the described bright structures (Video 3 , ESI $\dagger$ ). The principle of the 'FCS movie' is given in Fig. S8 (ESI + ).

To understand the diffusion behaviour of the abovedescribed structures, we utilized the 'FCS diffusion law' analysis implemented on ITIR-FCS that identifies confined diffusion from a plot of diffusion time versus the observation area. ${ }^{16}$
Details of the analysis are given in the ESI. $\nmid$ It was interesting to observe that the FCS diffusion law intercept remained close to zero until 15 min while the fluidity of the membrane increased during this time (Fig. 3D). Beyond this time, an increase in the intercept is associated with an overall decrease in $D$. The positive intercept indicates the formation of non-penetrable domains. This correlated well with the appearance of the bright clusters in the image sequence. Cytotoxicity assay using MTT also indicated cell death $(\sim 80 \%)$ after $14 \mathrm{~h}$ of incubation with $1 \mu \mathrm{M}$ hIAPP suggesting that the observed membrane destabilization is cytotoxic (Fig. S9, ESI $\dagger$ ). These observations suggest that the increase in fluidity of the plasma membrane by hIAPP induces a quantifiable biological response in the form of membrane reorganization not observed in artificial bilayers. Thus from the SLB and live cell membrane studies, we conclude that monomeric hIAPP interacts with membranes via dynamic carpet formation followed by lipid extraction in a concentration dependent manner (Fig. 3E).

Our results suggest that monomeric hIAPP, alone, can enhance membrane fluidity depending on its concentration and the membrane composition and also of the live plasma membranes leading to significant membrane reorganization. Based on these findings, we propose that monomeric hIAPP can present itself as a cytotoxic entity beyond a critical concentration; pre-aggregation per se may not be essential. However, we cannot exclude the possibility that hIAPP forms oligomers or aggregates during or after its interaction with the membrane. Nonetheless, amyloid plaques are implicated in degenerating pancreas of diabetes mellitus type II patients. We believe that our findings will prove valuable in the understanding of hIAPP mediated cellular damage in a new light and aid in the design of relevant therapeutic interventions.

\section{Notes and references}

1 P. Westermark, A. Andersson and G. T. Westermark, Phys. Rev., 2011, 91, 795-826.

2 N. B. Last, E. Rhoades and A. D. Miranker, Proc. Natl. Acad. Sci. U. S. A., 2011, 108, 9460-9465.

3 J. Sankaran, M. Manna, L. Guo, R. Kraut and T. Wohland, Biophys. J., 2009, 97, 2630-2639.

4 N. Bag, J. Sankaran, A. Paul, R. S. Kraut and T. Wohland, ChemPhysChem, 2012, 13, 2784-2794.

5 M. Magzoub and A. D. Miranker, FASEB J., 2012, 26, 1228-1238.

6 R. Soong, J. R. Brender, P. M. Macdonald and A. Ramamoorthy, J. Am. Chem. Soc., 2009, 131, 7079-7085.

7 P. Hammarstrom, R. Simon, S. Nystrom, P. Konradsson, A. Aslund and K. P. Nilsson, Biochemistry, 2010, 49, 6838-6845.

8 Y. A. Domanov and P. K. J. Kinnunen, J. Mol. Biol., 2008, 376, 42-54.

9 X.-B. Mao, C.-X. Wang, X.-K. Wu, X.-J. Ma, L. Liu, L. Zhang, L. Niu, Y.-Y. Guo, D.-H. Li, Y.-L. Yang and C. Wang, Proc. Natl. Acad. Sci. U. S. A., 2011, 108, 19605-19610.

10 Y. Gofman, T. Haliloglu and N. Ben-Tal, Nucleic Acids Res., 2012, 40, W358-W363.

11 S. M. Patil, S. Xu, S. R. Sheftic and A. T. Alexandrescu, J. Biol. Chem., 2009, 284, 11982-11991.

12 G. Drin and B. Antonny, FEBS Lett., 2010, 584, 1840-1847.

13 M. N. Melo, R. Ferre and M. A. Castanho, Nat. Rev. Microbiol., 2009, 7, 245-250.

14 P. Winckler, A. Cailler, R. Deturche, P. Jeannesson, H. Morjani and R. Jaffiol, Biochim. Biophys. Acta, 2012, 1818, 2477-2485.

15 J. Janson, R. H. Ashley, D. Harrison, S. McIntyre and P. C. Butler, Diabetes, 1999, 48, 491-498.

16 L. Wawrezinieck, H. Rigneault, D. Marguet and P. F. Lenne, Biophys. J., 2005, 89, 4029-4042. 